\title{
KRULL-GABRIEL DIMENSION AND THE MODEL-THEORETIC COMPLEXITY OF THE CATEGORY OF MODULES OVER GROUP RINGS OF FINITE GROUPS
}

\author{
GENA PUNINSKI, VERA PUNINSKAYA, AND CARLO TOFFALORI \\ ABstract. We classify group rings of finite groups over a field $F$ accord- \\ ing to the model-theoretic complexity of the category of their modules. \\ For instance we prove that, if $F$ contains a primitive cubic root of 1 , \\ then the Krull-Gabriel dimension of such rings is 0, 2, or undefined.
}

\section{INTRODUCTION}

There are two standard tools to measure a model-theoretic complexity of the category of modules over a given ring $R$. The first one is the KrullGabriel dimension which can be defined as the $m$-dimension of the lattice of all positive primitive formulae over $R$. In the model theory of modules this notion is tightly connected with the Cantor-Bendixson analysis of the Ziegler spectrum of $R$ (a topological space, whose points are indecomposable pure-injective $R$-modules), and hence with a classification of indecomposable pure-injective modules. In all known cases where the Krull-Gabriel dimension has been calculated, it is equal to the Cantor-Bendixson rank of the Ziegler spectrum, and it is an open question whether it is always the case.

The notion of the Krull-Gabriel dimension was introduced by Geigle [11] in the context of finite dimensional algebras over a field. In particular, he proved (see [11, Thm. 4.3]) that the Krull-Gabriel dimension of any tame hereditary finite dimensional algebra over a field is equal to 2 . It is also known from Auslander [1, Prop. 3.4] that the Krull-Gabriel dimension of a finite dimensional algebra $A$ (or any ring) is equal to zero if and only if $A$ is of finite representation type, and Baer (see [2, Cor. 3.7]) showed that the Krull-Gabriel dimension of any wild finite dimensional algebra is undefined. Furthermore, by Krause [17] (see also Herzog [14]) the KrullGabriel dimension of a finite dimensional algebra cannot be equal to 1 .

2000 Mathematics Subject Classification. 16D50,16S34,16G30.

Key words and phrases. Krull-Gabriel dimension, width, group rings of finite groups. The research of the first author is partially supported by NFS Grant DMS-0612720. 
Recently Schröer [28] and (independently) Burke and Prest [5] found, for every $n \geq 2$, an example of a (string) finite dimensional algebra with KrullGabriel dimension $n$. It is still an open question whether there exists a finite dimensional algebra with an infinite Krull-Gabriel dimension. Again, by Schröer (see also [21]), if $A$ is a finite dimensional non-domestic string algebra over a field, then the Krull-Gabriel dimension of $A$ is undefined, and there is a belief that every domestic finite dimensional string algebra has finite Krull-Gabriel dimension (see [22, 24]).

Besides its applications to the description of points and topology of the Ziegler spectrum, the (existence of) Krull-Gabriel dimension eradicates some 'strange' pure-injective modules. Namely, if the Krull-Gabriel dimension of a ring $R$ is defined, then every pure-injective $R$-module has an indecomposable direct summand, hence there is no superdecomposable pure-injective module over $R$.

Another more powerful tool to eliminate superdecomposable pure-injective modules is the width (of the lattice of all pp-formulae) over a ring $R$. Thus, if $R$ has a width, then no superdecomposable modules over $R$ occur, but the converse was proved by Ziegler only for countable rings (thus, in the case of finite dimensional algebras, for countable fields). Essentially less is known about this model-theoretic invariant of $R$. It is a kind of folklore (see [20, p. 282]) that every wild algebra does not have width. (For a discussion of a more subtle question of the existence of superdecomposable pure-injective modules over wild algebras see [16, Ch. 8]). Recently Puninski [23] pinpointed a class of tame finite dimensional algebras without width. Namely, he proved that every non-domestic finite dimensional string algebra does not have width. No example of a finite dimensional algebra with width, but without Krull-Gabriel dimension is known. For instance, it is not known whether all Ringel's canonical algebras (see [27]) do not have width (none of them has Krull-Gabriel dimension as observed by Prest [20, p. 276]).

Thus, it sounds useful to look at some natural classes of finite dimensional algebras (or rings) to measure their complexity using the Krull-Gabriel dimension or width. Unfortunately for many classes of rings that are at hands the model-theoretic complexity grows too rapidly. For instance, Puninski, Puninskaya and Toffalori [25] have recently shown that, if $R$ is the integral group ring of a (nontrivial) finite group, then the width of $R$ is undefined and hence (since $R$ is countable) there exists a superdecomposable pure-injective $R$-module. 
In this paper we investigate from this point of view group rings $F G$ of finite groups over a field $F$. By Maschke's theorem nothing interesting is happening if the characteristic of $F$ does not divide the order of $G$ (for instance, if the characteristic of $F$ is zero). Indeed, $F G$ is semisimple artinian in this case, hence the Krull-Gabriel dimension of $F G$ is zero. If $F$ is a field of characteristic $p$ and $p$ divides $|G|$, then (see $[8,64.1]$ ) the group ring $F G$ is of finite representation type if and only if a $p$-Sylow subgroup of $G$ is cyclic. This gives a description of group rings with Krull-Gabriel dimension zero. As we have already mentioned, the Krull-Gabriel dimension of $F G$ cannot be equal to 1 .

It is easily seen that, if $G=C(2)^{2}$ is the Klein group and the characteristic of $F$ is equal to 2, then the Krull-Gabriel dimension of $F G$ is equal to 2 (see Fact 4.3 below). Using a standard (induce and restrict) machinery we show (see Corollary 4.10) that the same is true for any finite group $G$ with $C(2)^{2}$ as a 2-Sylow subgroup and for a field $F$ of characteristic 2. The standard examples here are given by the alternating groups $A_{4}$ and $A_{5}$ over a field of characteristic 2 , but further groups with this property can be found among the simple groups $P S L_{2}(q)$ : for instance, $P S L_{2}(11)$ and $P S L_{2}(13)$ are such. In fact we completely characterize (see Corollary 4.16) finite simple groups $G$ such that the group ring $F G$ has Krull-Gabriel dimension 2: all these groups are in the $P S L_{2}(q)$ series and the characteristic of $F$ must be equal to 2 .

But, just as for integral group rings, a big gap arises in the possible values of Krull-Gabriel dimension for our group algebras. Namely, we will prove (see Theorem 4.11) that, if $F$ is a field and $G$ is a finite group not mentioned above, then in most cases the group ring $F G$ has no width, hence the KrullGabriel dimension is undefined; in particular, if $F$ is countable, then $F G$ possesses a superdecomposable pure-injective module. The only possible exceptions are generalized quaternion groups over a field of characteristic 2 without a primitive cubic root of unity.

The proof is straightforward when $F G$ is wild (see the above remarks), and it is well known that the remaining cases are but a few. Indeed, as we show below, it suffices to look at the group algebras $F G$ where $G$ is isomorphic either to $D(8)$ (the dihedral group) or to $Q(8)$ (the quaternion group) and $F$ is a field of characteristic 2 . We will handle $D(8)$ using a standard series of string modules, similarly to [23]. If $F$ contains a primitive 
cubic root of unity, a clever Dade's substitute (see [10]) will allow us to employ a similar approach in the case of $Q(8)$.

So the only case to be clarified, and the main difficulty we encounter, is when $G=Q(8)$ and $F$ is a field of characteristic 2 containing no primitive cubic root of unity (say, a finite field of two elements). The relations of $F Q(8)$ in this case seem to be too long to produce a natural series of string modules (to prove the non-existence of width). Still we believe that the width of $F Q(8)$ is undefined, and plan to overcome these difficulties in a future paper.

Our approach is based on [23], but we will also provide (and apply to our framework) a new very general condition under which a ring $R$ does not have width. Roughly speaking this condition is met when $R$ has two 'independent' dense chains of finitely presented modules with local endomorphism rings. To prove this we will exploit essentially the same idea as in [23]: a lattice freely generated by two dense chains does not have width.

In detail $\S \S 2-3$ are devoted to preliminaries and basic results on lattices of pp-formulae and dimensions, respectively. $\S 4$ deals with the complexity of modules over group algebras, and $\S 5$ introduces and illustrates the width criterion just mentioned.

To make this paper accessible for both model theorists and experts in the representation theory, we will introduce the basic notions using the language of both areas. However, the machinery used in the proofs is clearly biased towards the model theory of modules (reflecting mostly the authors background). Anyway we refer to [16], [20] and [32] for model theory of modules, to [12] and [31] for group theory and to [8], [9] and [30] for representation theory. Modules are assumed to be right; as we will see in the next section, this preference (right rather than left) does not affect our results.

\section{Preliminaries AND PP-Formulae.}

There are many ways to define a positive primitive formula (pp-formula) over a given ring $R$. Using functors one can say that a pp-formula is a finitely generated subfunctor of the forgetful functor $\operatorname{Hom}(R,-)$ in the category (mod- $R, \mathrm{Ab})$ of additive covariant functors, where $\bmod -R$ is the category of finitely presented $R$-modules and $\mathrm{Ab}$ is the category of abelian groups. Model-theoretic-wise the definition is as follows.

A pp-formula $\varphi(x)$ (in one free variable $x$ ) is a formula of the form $\exists \bar{y}(\bar{y} A=x \bar{b})$, where $\bar{y}=\left(y_{1}, \ldots, y_{k}\right)$ is a set of (bound) variables, $A$ is 
an $k \times l$ matrix over $R$, and $\bar{b}=\left(b_{1}, \ldots, b_{l}\right)$ is a row of elements of $R$. For instance $a \mid x$ (read ' $a$ divides $x$ ') is a divisibility formula $\exists y(y a=x)$. If $M$ is a module and $m \in M$, then one writes $M \models \varphi(m)$ ( $m$ satisfies $\varphi$ in $M)$, if there exists a tuple of elements $\bar{m}=\left(m_{1}, \ldots, m_{k}\right) \in M$ such that $\bar{m} A=m \bar{b}$. The set $\varphi(M)=\{m \in M|M| \varphi(m)\}$ is a subgroup of $M$ and is said to be a pp-subgroup of $M$. It may happen (if $R$ is noncommutative) that $\varphi(M)$ is not a submodule of $M$, but it is always a (left) submodule of $M$ over $S=\operatorname{End}(M)$. For instance, if $\varphi$ is a divisibility formula $a \mid x$, then $\varphi(M)=M a$.

If $f: M \rightarrow N$ is a morphism of modules and $M \models \varphi(m)$ for some pp-formula $\varphi$, then $N=\varphi(f(m))$, hence $f(\varphi(M)) \subseteq \varphi(N)$. Thus every pp-formula $\varphi(x)$ defines a functor $F_{\varphi} \in(\bmod -R, \mathrm{Ab})$ as follows. For every $M \in \bmod -R$ we set $F_{\varphi}(M)=\varphi(M)$ and, if $f: M \rightarrow N$ is a morphism of finitely presented modules, then $F(f)$ is the restriction of $f$ on $\varphi(M)$.

If $\varphi(x)$ and $\psi(x)$ are pp-formulae, we say that $\varphi$ implies $\psi$, written $\varphi \rightarrow \psi$, if for every (finitely presented) module $M, \varphi(M) \subseteq \psi(M)$. For instance, if $\varphi \doteq a \mid x$ and $\psi \doteq b \mid x, a, b \in R$, then $\varphi \rightarrow \psi$ if and only if $a \in R b$. It follows that $\varphi \rightarrow \psi$ if and only if $F_{\varphi}$ is a subfunctor of $F_{\psi}$. Now we identify ppformulae that define the same functor. Namely, we say that $\varphi$ is equivalent to $\psi$, if $\varphi \rightarrow \psi \rightarrow \varphi$, that is, $\varphi(M)=\psi(M)$ for every module $M$. Then the lattice of pp-formulae $L_{R}$ is the lattice of finitely generated subfunctors of the functor $(R,-) \in(\bmod -R, \mathrm{Ab})$ with + and $\cap$ as operations.

On the level of pp-formulae these operations are identified as follows. If $\varphi$ and $\psi$ are pp-formulae, then their meet is a conjunction $\varphi \wedge \psi$, and joint (that is, sum) of $\varphi$ and $\psi$ can be defined as the pp-formula $\theta(x) \doteq$ $\exists z(\varphi(z) \wedge \psi(x-z))$.

A third way to look at pp-formulae is through pointed modules. A pointed module $(M, m)$ is a module $M$ with a distinguished element $m \in M$. A morphism of pointed modules is a morphism $f: M \rightarrow N$ such that $f(m)=n$. One can also think of a pointed module $(M, m)$ as a morphism $g_{M}: R \rightarrow M$ such that $g_{M}\left(1_{R}\right)=m$ (where $1_{R}$ denotes the unity of $R$ ). From this point of view a pointed morphism is a morphism $f: M \rightarrow N$ such that $f g_{M}=g_{N}$.

To see the way all this is connected with pp-formulae, let $(M, m)$ be a pointed module such that $M$ is finitely presented. Then (see [20, Prop. 8.4]) there is a pp-formula $\varphi(x)$ such that $(M, m)$ is a free realization of $\varphi$, that is, $m \in \varphi(M)$ and for every pp-formula $\psi, m \in \psi(M)$ yields $\varphi \rightarrow \psi$ (thus a free 
realization is a 'minimal' realization of $\varphi$ ). Furthermore, $\varphi$ is uniquely determined up to equivalence, and (again by [20, Prop. 8.4]) every pp-formula has a finitely presented free realization.

Thus the lattice $L_{R}$ can be considered as a set of equivalence classes of (pointed) morphisms between finitely presented pointed modules. Here, two pointed modules $(M, m)$ and $(N, n)$ are said to be equivalent, if there are pointed morphisms $f: M \rightarrow N$ and $g: N \rightarrow M$ (such that $f(m)=n$ and $g(n)=m)$. The operations of the lattice $L_{R}$ obtain a nice description on this level. Namely, if $(M, m)$ corresponds to $\varphi$ and $(N, n)$ corresponds to $\psi$, then $(M \oplus N,(m, n))$ will correspond to $\varphi+\psi$. To get $\varphi \wedge \psi$ is more tricky: one should form a pushout of the corresponding morphisms $f: R \rightarrow M$ and $g: R \rightarrow N$ which gives a finitely presented module $K=M \oplus N / \operatorname{im}(f,-g)$. If $k$ denotes the common coset of $(m, 0)$ and $(0, n)$, then $(K, k)$ will be a free realization of $\varphi \wedge \psi$ (for more on this see [18, Ch. 7]).

$L_{R}$ is a modular lattice. Furthermore $L_{R}$ has the largest element $1\left(L_{R}\right)$ given by the (equivalence class of) pp-formula $x=x$, and the least element $0\left(L_{R}\right)$ given by the pp-formula $x=0$. On the level of morphisms they correspond to the identity morphism from $R$ into $R$ and to the zero morphism from $R$ onto 0 .

There are two subtleties to be taken into account looking at the above definition. Firstly it has been introduced for right modules over $R$, so we should be talking about right pp-formulas. A similar definition is possible on the left, so we get a lattice of left pp-formulae over $R$. Fortunately, by [20, Ch. 8] (which is just a reincarnation of Auslander's duality) these lattices are anti-isomorphic. So for the purpose of this paper (say, calculating different dimension, see below) the side does not make any difference. Therefore we use $L_{R}$ to denote either of these lattices.

Secondly we have considered only pp-formulae in one free variable. A very similar definition is possible for pp-formulae $\varphi(\bar{x})$ in any number of free variables $\bar{x}=\left(x_{1}, \ldots, x_{k}\right)$. On the functorial level they correspond to finitely generated subfunctors of the forgetful functor $\operatorname{Hom}\left(R^{k},-\right) \in(\bmod -R, \mathrm{Ab})$. We can also identify these pp-formulae with pointed modules $(M, \bar{m})$, where $\bar{m}=\left(m_{1}, \ldots, m_{k}\right)$ is a tuple of elements of $M$, or with morphisms $f: R^{k} \rightarrow$ $M$ such that $f\left(e_{i}\right)=m_{i}\left(e_{i}\right.$ is the $i$ th unity of $\left.R^{k}\right)$. A pp-formula $\varphi$ in $k$ free variables defines a subgroup of $M^{k}$ consisting of all $k$-tuples $\bar{m}$ satisfying $\varphi$, and we will still denote this subgroup by $\varphi(M)$. As before, identifying equivalent pp-formulae we obtain a lattice $L_{R}^{k}$. According to this notation 
$L_{R}^{1}$ is just $L_{R}$. Most questions in the model theory of modules can be solved on the level of $L_{R}^{1}$, but sometimes one has to evoke the $L_{R}^{k}$.

\section{Dimensions}

There is a general way of introducing an (ordinal valued) dimension of a modular lattice with respect to any class of modular lattices (see [20, Sec. 10.2]). In the model theory of modules just two of them are in a common use. The former, referring to the 2 -element chain, is $m$-dimension, and the latter, corresponding to the set of all chains, is breadth. There is one more important dimension which does not fall completely in this description, although it is connected with breadth. It is the (Ziegler's) width of the lattice which plays a dominant role in this paper. Let us (following [20, Sect. 10.2]) briefly recall these notions.

Let $L$ be a modular lattice with with bottom element $0(L)$ and top element $1(L)$. We will define the $m$-dimension of $L, \operatorname{mdim}(L)$. To start with, we set $\operatorname{mdim}(L)=-1$ if and only if $|L|=1$, that is, $0(L)=1_{L}$. Otherwise $|L|>1$, hence $\operatorname{mdim}(L) \geq 0$.

Let $\sim_{f}$ be a congruence on a modular lattice that identifies intervals of finite length. By induction on ordinals we define an ascending chain of congruences $\sim_{\alpha}$ on $L$ and the corresponding chain of factor lattices $L_{\alpha}=$ $L / \sim_{\alpha}$.

If $\alpha=0$, then $\sim_{\alpha}$ is the trivial congruence, hence $L_{0}=L / \sim_{0}$ is isomorphic to $L$. If $\alpha$ is a limit ordinal, then set $\sim_{\alpha}=\cup_{\beta<\alpha} \sim_{\beta}$, and let $L_{\alpha}$ be the corresponding factor lattice of $L$.

Suppose that $\alpha=\beta+1$ is such that $\sim_{\beta}$ has been already defined, and let $\pi_{\beta}: L \rightarrow L_{\beta}$ be the corresponding projection. Then the congruence $\sim_{\alpha+1}$ is defined to be the preimage, via $\pi_{\beta}$, of the congruence $\sim_{f}$ on $L_{\beta}$.

By cardinality arguments, the ascending chain of congruences $\sim_{\alpha}$ on $L$ will stabilize. Thus there are two cases to consider. If this chain stabilizes on certain $\alpha$ such that $0(L)$ is not $\sim_{\alpha}$-equivalent to $1(L)$ (hence $L_{\alpha}$ has at least two elements) we say that the $m$-dimension of $L$ is undefined, or also that $\operatorname{mdim}(L)=\infty$. If this is not the case then, since $0(L) \neq 1(L)$, there exists a successor ordinal $\alpha=\beta+1 \operatorname{such}\left|L_{\beta}\right|>1$, but $\left|L_{\alpha}\right|=1$, and we say that the $m$-dimension of $L$ is equal to $\beta, \operatorname{mdim}(L)=\beta$.

Thus the $m$-dimension of $L$ is zero if and only if $L$ is a finite lattice with $0(L) \neq 1(L)$. Furthermore, if $\omega$ is the usual ordering of natural numbers and $\omega^{*}$ denotes its reverse ordering, then the chain $L=\omega \triangleleft \omega^{*}$ ( $\omega^{*}$ on the 
top of $\omega$ ) has $m$-dimension 1. Indeed, $\sim_{1}$ identifies all finite length intervals of $L$, hence glues together all the elements of $\omega$, and also those of $\omega^{*}$, but still separates the elements of $\omega$ and those of $\omega^{*}$. Thus $L_{1}$ is a two-element lattice, and $L_{2}$ is a trivial lattice, hence $\operatorname{mdim}(L)=1$. It can be also checked that the $m$-dimension of a lattice $L$ is undefined if and only if $L$ contains a subchain isomorphic to the ordering $(\mathbb{Q}, \leq)$ of the rationals.

An important property of the $m$-dimension is that, if $L^{\prime}$ is a sublattice (even with different bottom and top elements) or a factor lattice of $L$, then $\operatorname{mdim}\left(L^{\prime}\right) \leq \operatorname{mdim}(L)$.

Lemma 3.1. (see [18, L. B6]) If $L=L_{1} \times \ldots \times L_{k}$, then $\operatorname{mdim}(L)=$ $\max _{i} \operatorname{mdim} L_{i}$.

Proof. Since each $L_{i}$ is embeddable in $L, \operatorname{mdim}\left(L_{i}\right) \leq \operatorname{mdim}(L)$ for every $i$, hence $\max _{i} \operatorname{mdim}\left(L_{i}\right) \leq \operatorname{mdim}(L)$.

To prove the reverse inequality we may argue by induction, and hence assume that $k=2$. Suppose that $\operatorname{mdim}\left(L_{1}\right)=\alpha$ and $\operatorname{mdim}\left(L_{2}\right)=\beta$ such that $\alpha \geq \beta$. Consider the following chain in $L=L_{1} \times L_{2}: 0(L)=$ $\left(0\left(L_{1}\right), 0\left(L_{2}\right)\right)<\left(1\left(L_{1}\right), 0\left(L_{2}\right)\right)<\left(1\left(L_{1}\right), 1\left(L_{2}\right)\right)=1(L)$. Clearly the interval $\left[\left(0\left(L_{1}\right), 0\left(L_{2}\right)\right) ;\left(1\left(L_{1}\right), 0\left(L_{2}\right)\right)\right]$ is isomorphic to $L_{1}$, hence it got collapsed on the stage $\alpha$ (as we have already noticed, there is no difference whether we run $\sim_{\alpha}$ analysis in $L$ or in any of its sublattices). Similarly the interval $\left[\left(1\left(L_{1}\right), 0\left(L_{2}\right)\right) ;\left(1\left(L_{1}\right), 1\left(L_{2}\right)\right)\right]$ is isomorphic to $L_{2}$, hence it got collapsed at the stage $\alpha$. It follows that $0(L) \sim_{\alpha} 1(L)$ in $L$, therefore $\operatorname{mdim}(L) \leq \alpha$.

It is easily seen from the definition that the $m$-dimension does not change its value if one switch from $L$ to its dual lattice $L^{*}$. We single out this fact for future applications.

Fact 3.2. If $L$ is a modular lattice with bottom element $0(L)$ and top element $1(L)$, then $\operatorname{mdim}(L)=\operatorname{mdim}\left(L^{*}\right)$.

Now let us deal with breadth. Let $L$ be a modular lattice with bottom element $0(L)$ and top element $1(L)$, and let $\sim_{l}$ be a congruence on $L$ that identifies intervals which are chains (strictly speaking, it is a congruence generated by these intervals, that is, $a \sim_{l} b$ if and only if there is a chain $a \wedge b=c_{0}<c_{1}<\ldots<c_{k}=a+b$ such that each interval $\left[c_{i}, c_{i+1}\right]$ in $L$ is a chain). The breadth of $L, \operatorname{br}(L)$, is defined in the same manner as $m$-dimension, but using the congruence $\sim_{l}$ instead of $\sim_{f}$ at each stage.

By this definition, it follows again that $\operatorname{br}(L)=-1$ if and only if $|L|=1$; also $\operatorname{br}(L)=0$ if and only if there is a chain $0=c_{0}<\ldots<c_{k}=1$ in $L$ such 
that each interval $\left[c_{i}, c_{i+1}\right]$ in $L$ is a chain. Thus the following lemma has the same proof as Lemma 3.1.

Lemma 3.3. If $L=L_{1} \times \ldots \times L_{k}$, then $\operatorname{br}(L)=\max _{i} \operatorname{br}\left(L_{i}\right)$.

The usage of breadth of a lattice is quite restricted because in most cases it can be replaced by a (less smooth) invariant called width. However, Lemma 3.3 is not true for width, so we have to use breadth in some proofs. On the other hand most results about superdecomposable pure-injective modules are formulated in terms of width, hence we have to introduce this notion too.

Let $L$ be a modular lattice with bottom element $0(L)$ and top element $1(L)$. We define the width of $L, \operatorname{wd}(L)$, by induction on ordinals. We set $\operatorname{wd}(L)=-1$ if and only if $|L|=1$, and, if $\alpha$ is a limit ordinal, then define $\operatorname{wd}(L) \geq \alpha$ if $\operatorname{wd}(L) \geq \beta$ for every $\beta<\alpha$ (as has been already decided). Suppose that $\alpha=\beta+1$. Then one puts $\operatorname{wd}(L) \geq \alpha$ if there exist $a, b \in L$ such that both intervals $[a, a+b]$ and $[b, a+b]$ have width $\geq \beta$.

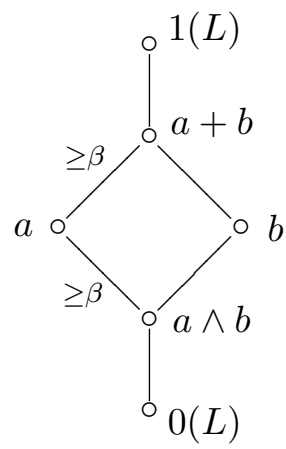

By modularity, it is the same as $\operatorname{wd}([a \wedge b, a]) \geq \beta$ and $\operatorname{wd}([a \wedge b, b]) \geq \beta$. For instance, $\operatorname{wd}(L)=0$ if and only if $L$ is a chain, but the direct product of two nontrivial chains has width 1 (and breadth 0 ). As one can easily see from the definition, the breadth does not exceed the width. In fact both dimensions coexist.

Fact 3.4. $[20$, L. 10.7] Let $L$ be a modular lattice with bottom element $0(L)$ and top element $1(L)$. Then $\operatorname{br}(L) \leq \operatorname{wd}(L)<\omega^{\operatorname{br}(L)+1}$.

Note that the $\sim_{\alpha}$ analysis for breadth runs at least as fast as a similar analysis for $m$-dimension. Thus we obtain the following:

Corollary 3.5. Let $L$ be a modular lattice with $0(L)$ and $1(L)$. Then $\operatorname{mdim}(L) \geq \operatorname{br}(L)$. In particular, if the $m$-dimension of $L$ is defined, then the breadth of $L$ is defined, therefore the width of $L$ is defined. 
Our main interest will be to calculate the aforementioned dimensions for lattices of pp-formulae over various given rings. Let us explain why this problem is important.

Let $R$ be a ring. Then the Krull-Gabriel dimension of $R, \operatorname{KG}(R)$, is the $m$-dimension of the lattice of all pp-formulae (in one variable) over $R$. Again, two precautions should be taken. Firstly we should check that this definition does not depend on the side. But, as we have already mentioned, the lattice of left pp-formulae over $R$ is anti-isomorphic to the lattice of right pp-formulae over $R$, hence (see Fact 3.2) both lattices have the same $m$ dimension. Secondly we will see below that this definition does not depend on the number of variables in pp-formulae (this can be also extracted from [18, Prop. 7.2]).

If $M$ is a module over $R$, then the set of pp-subgroups of $M$ forms a lattice $L(M)$. Moreover, the map $\varphi \rightarrow \varphi(M)$ defines a morphism of lattices $L_{R} \rightarrow L(M)$ which is onto. We say that a module $M$ is big, if this map is an isomorphism. Big modules exist over any ring.

Fact 3.6. (see [20, Cor. 8.17]) Suppose that $M$ is a direct sum of all isomorphism types of finitely presented $R$-modules (or $M$ has this module as a direct summand). Then $M$ is big.

We may introduce a similar lattice $L^{k}(M)$ of subgroups of $M^{k}$ defined by pp-formulae in $k$ free variables. According to this notation $L^{1}(M)$ is just $L(M)$. As above, we obtain an onto morphism of lattices $L_{R}^{k} \rightarrow L^{k}(M)$, which is an isomorphism if $M$ is a big module.

Let $\varphi(x, y)$ be a pp-formula (in two free variables). Define $\varphi_{1}(x) \doteq$ $\exists y \varphi(x, y)$ and $\varphi_{2}(y) \doteq \varphi(0, y)$. Thus $\varphi_{1}$ and $\varphi_{2}$ are both pp-formulas in one variable.

Lemma 3.7. If $M$ is a module, then the map $\varphi(M) \rightarrow\left(\varphi_{1}(M), \varphi_{2}(M)\right)$ defines an embedding of lattices $L^{2}(M) \rightarrow L(M) \times L(M)$.

In particular, the map $\varphi \rightarrow\left(\varphi_{1}, \varphi_{2}\right)$ defines an embedding of $L_{R}^{2}$ into $L_{R} \times L_{R}$

Proof. The latter claim follows directly from the former, as soon as $M$ is chosen to be big (see Fact 3.6).

Concerning the former claim, it is straightforward to check that the map is a morphism of lattices. Indeed, it suffices to prove that, if $\varphi, \psi$ are ppformulae in two variables, then $\varphi(M) \subseteq \psi(M)$ implies $\varphi_{i}(M) \subseteq \psi_{i}(M)$ for every $i$. Suppose that $m \in \varphi_{1}(M)$. Then $M \models \exists y \varphi(m, y)$, hence there 
exists $n \in M$ such that $M \models \varphi(m, n)$. By the assumption, $M \models \psi(m, n)$, hence $M \models \psi_{1}(m)$, as desired. The proof that $\varphi_{2}(M) \subseteq \psi_{2}(M)$ is similar.

Now we check that this map is an embedding. Otherwise we may assume that $\varphi(M) \subset \psi(M)$ for some pp-formulae $\varphi$ and $\psi$ in two variables such that $\varphi_{1}(M)=\psi_{1}(M)$ and $\varphi_{2}(M)=\psi_{2}(M)$. Choose $m, n \in M$ such that $M \models \psi(m, n) \wedge \neg \varphi(m, n)$. From $M \models \psi(m, n)$ it follows that $M \models \psi_{1}(m)$. By the assumption, $M \models \varphi_{1}(m)$, therefore $M \models \varphi\left(m, n^{\prime}\right)$ for some $n^{\prime} \in M$. Since $\varphi(M) \subseteq \psi(M)$, we conclude that $M \models \psi\left(m, n^{\prime}\right)$. Subtracting this from $M=\psi(m, n)$, we obtain $M \models \psi\left(0, n-n^{\prime}\right)$, that is, $M \models \psi_{2}\left(n-n^{\prime}\right)$. By the assumption, $M=\varphi_{2}\left(n-n^{\prime}\right)$, that is, $M=\varphi\left(0, n-n^{\prime}\right)$. Adding this with $M \models \varphi\left(m, n^{\prime}\right)$, we conclude that $M \models \varphi(m, n)$, a contradiction.

It is easy to extend the above construction and to embed $L_{R}^{k}$ into the direct product of $k$ copies of $L_{R}$ for every $k$; in the same way, for every module $M$, on gets an embedding from $L^{k}(M)$ into the direct product of $k$ copies of $L(M)$.

Proposition 3.8. (cp. [18, Prop. 7.2]) For every ring $R$ we have $\operatorname{mim}\left(L_{R}^{k}\right)=$ $\operatorname{mdim}\left(L_{R}\right)$. Thus the Krull-Gabriel dimension of $R$ is equal to the $m$ dimension of the lattice of all pp-formulae in $k$ free variables.

If $M$ is a module, then $\operatorname{mdim}\left(L^{k}(M)\right)=\operatorname{mdim}(L(M))$.

Proof. Using induction we may assume that $k=2$. Then the result follows from Lemma 3.1 and Lemma 3.7.

By Lemma 3.3, similar arguments apply to the breadth.

Proposition 3.9. For any ring $R$ and any $k, \operatorname{br}\left(L_{R}^{k}\right)=\operatorname{br}\left(L_{R}\right)$. If $M$ is an R-module, then $\operatorname{br}\left(L^{k}(M)\right)=\operatorname{br}(L(M))$.

We apply this proposition as follows.

Corollary 3.10. For any ring $R$, if $\operatorname{wd}\left(L_{R}\right)$ is defined, then $\operatorname{wd}\left(L_{R}^{k}\right)$ is defined.

Proof. Suppose that $\mathrm{wd}\left(L_{R}\right)=\alpha$. By Fact 3.4, $\operatorname{br}\left(L_{R}\right) \leq \alpha$. By Proposition 3.9 we conclude that $\operatorname{br}\left(L_{R}^{k}\right) \leq \alpha$, hence (by Fact 3.4 again) $\operatorname{wd}\left(L_{R}^{k}\right)<$ $\omega^{\alpha+1}$.

Question 3.11. Let $R$ be a ring. Is it always true that $\operatorname{wd}\left(L_{R}\right)=\operatorname{wd}\left(L_{R}^{2}\right)$ ?

If $M$ is a module, and $\varphi$ is a pp-formula, then $\varphi\left(M^{(I)}\right)=\varphi(M)^{(I)}$. This readily implies that $L(M) \cong L\left(M^{(I)}\right)$, in particular these lattices 
have the same $m$-dimension and breadth. If $N$ is another module, then $\varphi(M \oplus N)=\varphi(M) \oplus \varphi(N)$, hence $L(M \oplus N)$ is embeddable in $L(M) \times L(N)$. By Lemma 3.1 we obtain

Corollary 3.12. (see [18, Prop. 7.6]) If $M$ and $N$ are modules and $I$ is a set, then $\operatorname{mdim}\left(L\left(M^{(I)} \oplus N\right)\right)=\max (\operatorname{mdim}(L(M)), \operatorname{mdim}(L(N)))$.

\section{Group algebras}

In this section we collect some (well known) facts about finite groups and their group rings over a field and apply them to calculate the model-theoretic complexities of these rings.

Recall that a (finite) group $G$ is said to be a $p$-group (for $p$ a prime), if the order of every element of $G$ is a power of $p$. Let $C\left(p^{k}\right)$ denote a cyclic $\left(p\right.$-)group consisting of $p^{k}$ elements.

Fact 4.1. (see $[8,6.10$, p. 19], for instance) If $G$ is a finite p-group then either $G$ is cyclic or $C(p)^{2}$ is a factor group of $G$.

For a definition of tame and wild finite dimensional algebras see [30, Sec. 14.4]. Here is a typical example of wildness.

Fact 4.2. (see [30, Thm. 14.17]) If $F$ is a field of characteristic $p>2$, then $F C(p)^{2}$ is a wild algebra. Consequently, if $G$ is a noncyclic p-group, then $F G$ is a wild algebra.

On the other hand the ring $F C(p)$, where $F$ is a field of characteristic $p$, is isomorphic to the (truncated) ring of polynomials $F\left[X \mid X^{p}=1\right]$, hence is of finite representation type.

For $p=2$ the situation is more complicated. First of all there exists a group algebra of Krull-Gabriel dimension 2.

Fact 4.3. If $F$ is a field of characteristic 2 and $G=C(2)^{2}$ is the Klein group, then FG has Krull-Gabriel dimension 2.

This should be folklore and in discussed for instance in $[19, \S 4]$. Anyway let us sketch briefly a proof.

Proof. Let $g$ and $h$ be generators of $C(2)^{2}$. If $X=1+g$ and $Y=1+h$, then $R=F C(2)^{2}$ is the (4-dimensional) algebra $F\left[X, Y \mid X^{2}=Y^{2}=\right.$ $0]$. As every group algebra of finite group, $R$ is self-injective with the (1dimensional) socle spanned by $X Y$. 
Therefore (see [16, Prop. 8.69]) every $R$-module is a direct sum of a free module $R^{(I)}$ and a module over $R / \operatorname{soc}(R) \cong F\left[X, Y \mid X^{2}=Y^{2}=X Y=0\right]$. It is easily seen (via a reduction to the Kronecker algebra, see [16, Ch. 8]) that the last algebra has Krull-Gabriel dimension 2. If $M$ is a big module over $R$, then $M=R^{(I)} \oplus K$, and we may assume that $K$ is a big $R / \operatorname{soc}(R)$ module. Then $\operatorname{mdim}(L(K))=2$, and $\operatorname{mdim}(L(R))=0$, since $R$ is of finite length. By Corollary 3.12, we obtain $\operatorname{mdim}(L(M))=\operatorname{mdim}(L(K))=2$, as desired.

Recall (see [30, p. 293]) that the following is a standard representation of dihedral groups:

$$
D_{m}=\left\langle g, h \mid g^{2}=h^{2^{m}}=1, h g h=g\right\rangle, m \geq 1 .
$$

For instance $D_{1}$ is isomorphic to the Klein group and $D_{2}$ is the dihedral group $D(8)$ of order 8 .

Similarly the semidihedral groups are given as

$$
S_{m}=\left\langle g, h \mid g^{2}=h^{2^{m}}=1, h g=g h^{2^{m-1}-1}\right\rangle, m \geq 3,
$$

and (generalized) quaternion group are represented as

$$
Q_{m}=\left\langle g, h \mid g^{2}=h^{2^{m-1}}, g^{4}=1, h g h=g\right\rangle, m \geq 2 .
$$

For instance $Q_{2}$ is the quaternion group $Q(8)$ of order 8 .

The following is an important dichotomy for 2-groups.

Fact 4.4. ([12, 5.4.5] and before that) Let $G$ be a (finite) 2-group which is not cyclic. Then either a) $G$ has $C(4) \times C(2)^{2}$ or $C(2)^{3}$ as a factor group, or b) $G$ is a dihedral, semidihedral or generalized quaternion group.

If $G$ is a dihedral with $m \geq 2$ or semidihedral, then $G$ contains $D(8)$ as a subgroup. If $G$ is generalized quaternion, then $G$ contains $Q(8)$ as a subgroup.

In fact all algebras in a) are wild.

Fact 4.5. (see [30, Thm. 14.17]) If $F$ is a field of characteristic 2 and $G=$ $C(4) \times C(2)^{2}$ or $G=C(2)^{3}$, then the group algebra $F G$ is wild. Therefore the same is true if $G$ has one of this group as a factor group.

Dihedral, semidihedral and generalized quaternion algebras are tame (see [3]), but we do not use this result in the paper. 
To make further reductions we recall some basic facts about induced modules.

Let $S$ be a subgroup of a group $G$ and let $M$ be an $F S$-module. Then the $F G$-module $M^{G}=M \otimes_{F S} F G$ is said to be induced by $M$. On the other hand if $N$ is an $F G$-module then, restricting scalars to $F S$, we obtain a (restricted) module $N_{S}$ over $F S$.

The following fact describes what is happening if we apply these operations twice.

Fact 4.6. ([9, 10.A and 19.5.(ix) $])$

1) If $M$ is an FS-module, then $M$ is a direct summand of $\left(M^{G}\right)_{S}$.

2) If the characteristic of $F$ does not divide $|G: S|$ and $N$ is an $F G$ module, then $N$ is a direct summand of $\left(N_{S}\right)^{G}$.

In most sources (such as [9]) these results are formulated in the framework of finitely generated modules. But one can easily check that their proof goes through for all modules.

Now we are in a position to give a first application. Clearly, if $L^{\prime}$ is a sublattice of a lattice $L$, then $\operatorname{wd}\left(L^{\prime}\right) \leq \operatorname{wd}(L)$.

Proposition 4.7. Let $G$ be a finite group, $S$ be a subgroup of $G$ and let $F$ be a field. If $\operatorname{wd}\left(L_{F S}\right)$ is undefined, then $\operatorname{wd}\left(L_{F G}\right)$ is undefined. If $\operatorname{KG}(F S)$ is undefined, then $\operatorname{KG}(F G)$ is undefined.

Proof. Let $M$ be a big module over $F S$, hence $L(M)=L_{F S}$. It follows that $\operatorname{wd}\left(L_{F S}\right)=\operatorname{wd}(L(M))$, hence $\operatorname{wd}(L(M))=\infty$. By Fact 4.6, $M$ is a direct summand of $F S$-module $N=\left(M^{G}\right)_{S}$. It follows that $\operatorname{wd}(L(M)) \leq$ $\operatorname{wd}(L(N))$, hence $\operatorname{wd}(L(N))=\infty$. But clearly the lattice of pp-subgroups of $N$ (as an FS-module) is a sublattice of the lattice of pp-subgroups of $M^{G}$ (as an $F G$-module). Then $\operatorname{wd}(L(N)) \leq \operatorname{wd}\left(L\left(M^{G}\right)\right) \leq \operatorname{wd}\left(L_{F G}\right)$, therefore $\operatorname{wd}\left(L_{F G}\right)=\infty$.

The proof for the Krull-Gabriel dimension is similar.

Let $S$ be a subgroup of a finite group $G$ and let $g_{1}, \ldots, g_{k}$ be representatives of left cosets of $G$ modulo $S$. If $M$ is an $F S$-module, then every element $m$ of the $F G$-module $M^{G}$ can be uniquely written as $m_{1} \otimes g_{1}+\ldots+m_{k} \otimes g_{k}$. For $h \in G$ and $1 \leq i \leq k$, find $h^{\prime} \in H$ and $1 \leq j \leq k$ such that $g_{i} h=h^{\prime} g_{j}$; this implies $\left(m_{i} \otimes g_{i}\right) \cdot h=m_{i} h^{\prime} \otimes g_{j}$, which determines the $F G$-module 
structure. Using this representation, one can easily construct for every ppformula $\varphi(x)$ over $F G$ a pp-formula $\varphi^{*}\left(x_{1}, \ldots, x_{k}\right)$ over $F S$ such that

$$
M^{G} \models \varphi(m) \quad \text { if and only if } \quad M \models \varphi^{*}\left(m_{1}, \ldots, m_{k}\right)
$$

(and this definition does not depend on $m$ and $M$ - see [25, p. 53] for example).

Proposition 4.8. 1) The map $\varphi \rightarrow \varphi^{*}$ defines an embedding of lattices $L\left(M^{G}\right) \rightarrow L^{k}(M)$.

2) If the characteristic of $F$ does not divide $|G: S|$, then this map gives rise to an embedding of lattices $L_{F G} \rightarrow L_{F S}^{k}$.

Proof. 1) First we check that this map is a morphism of lattices. Suppose that $\varphi\left(M^{G}\right) \subseteq \psi\left(M^{G}\right)$ for some pp-formulae $\varphi, \psi$ over $F G$, and we have to verify that $\varphi^{*}(M) \subseteq \psi^{*}(M)$.

Suppose that $M \models \varphi^{*}\left(m_{1}, \ldots, m_{k}\right)$ for some $m_{1}, \ldots, m_{k} \in M$ and set $m=m_{1} \otimes g_{1}+\cdots+m_{k} \otimes g_{k} \in M^{G}$. Then, by $(*), M^{G} \models \varphi(m)$, therefore, by the assumption, $M^{G} \models \psi(m)$. Applying $(*)$ again, we obtain $M \models$ $\psi^{*}\left(m_{1}, \ldots, m_{k}\right)$, as desired.

Now we check that this map is an embedding. Otherwise $\varphi\left(M^{G}\right) \subset$ $\psi\left(M^{G}\right)$ for some pp-formulae $\varphi, \psi$ over $F G$ such that $\varphi^{*}(M)=\psi^{*}(M)$. Choose $m=m_{1} \otimes g_{1}+\cdots+m_{k} \otimes g_{k} \in \psi\left(M^{G}\right) \backslash \varphi\left(M^{G}\right)$. Then, by $(*)$, we obtain $M \models\left(\varphi^{*} \wedge \neg \psi^{*}\right)\left(m_{1}, \ldots, m_{k}\right)$, a contradiction.

2) First we prove that there exists a big $F G$-module $N$ such that $N_{S}$ is a big $F S$-module. Indeed, using Fact 3.6, choose a big $F S$-module $M$. If $K$ is a big $F G$-module, then the same is true for $N=M^{G} \oplus K$. By Fact 4.6, $M$ is a direct summand of $\left(M^{G}\right)_{S}$. Then $M$ is a direct summand of $N_{S}$, hence $N_{S}$ is a big $F S$-module.

So we assume that $N$ is a big $F G$-module such that $N_{S}$ is a big $F S$ module. By Fact 4.6, $N$ is a direct summand of $\left(N_{S}\right)^{G}$. By what we have already proved, the map $\varphi \rightarrow \varphi^{*}$ defines an embedding of lattices $L\left(\left(N_{S}\right)^{G}\right) \rightarrow L^{k}\left(N_{S}\right)$. Since the $F G$-module $\left(N_{S}\right)^{G}$ contains $N$ as a direct summand, it is big, hence $L\left(\left(N_{S}\right)^{G}\right)=L_{F G}$. Similarly, since $N_{S}$ is a big $F S$-module, $L^{k}\left(N_{S}\right)=L_{F S}^{k}$. Thus we obtained an embedding from $L_{F G}$ into $L_{F S}^{k}$, as desired.

Corollary 4.9. Let $F$ be a field of characteristic $p$ and let $S \subseteq G$ be finite groups such that $p$ does not divide $|G: S|$. Then $\operatorname{KG}(F S)=\operatorname{KG}(F G)$. 
Proof. Arguing as in Lemma 4.7, it is always the case that $\operatorname{KG}(F S) \leq$ $\mathrm{KG}(F G)$.

If $p$ does not divide $|G: S|$ then, by Proposition 4.8, there is an embedding $L_{F G} \rightarrow L_{F S}^{k}$, hence $\mathrm{KG}(F G) \leq \operatorname{mdim}\left(L_{F S}^{k}\right)=\mathrm{KG}(F S)$ by Proposition 3.8.

Using Fact 4.3, we immediately obtain.

Corollary 4.10. Let $F$ be a field of characteristic 2 and let $G$ be a finite group such that a 2-Sylow subgroup of $G$ is isomorphic to $C(2)^{2}$. Then $\mathrm{KG}(F G)=2$.

A simplest example of this situation is the group $A_{4}$. Indeed, $\left|A_{4}\right|=12=$ $2^{2} \cdot 3$, hence it has a 2 -Sylow subgroup consisting of 4 elements. In fact, the (normal) subgroup generated by permutations (12)(34) and (14)(23) is isomorphic to $C(2)^{2}$. If $G=A_{5}$, then $\left|A_{5}\right|=60=2^{2} \cdot 3 \cdot 5$, hence (the same) group $C(2)^{2}$ is a 2-Sylow subgroup of $A_{5}$ (but in this case it is not normal). It follows that, over a field $F$ of characteristic 2, both algebras $F A_{4}$ and $F A_{5}$ have Krull-Gabriel dimension 2. At the end of this section we will give more examples of group rings with Krull-Gabriel dimension 2.

If $F$ is a field of characteristic $p$ and $G$ is a finite group such that $p$ does not divide $|G|$, then the group ring $F G$ is semisimple artinian, hence $\operatorname{KG}(F G)=0$. Thus we concentrate on the case of prime characteristic $p$ dividing $|G|$.

If $A$ is a wild finite dimensional algebra, then (see [20, Thm. 13.7]) the width of $A$ is undefined.

Theorem 4.11. Let $F$ be a field of characteristic $p$ and let $G$ be a finite group such that $p$ divides $|G|$. Then

1) $\operatorname{KG}(F G)=0$ if and only if a p-Sylow subgroup of $G$ is cyclic.

Suppose that either the characteristic of $F$ is not equal to 2, or $F$ contains a primitive cubic root of unity, or a 2-Sylow subgroup of $G$ is not isomorphic to a generalized quaternion group. Then

2) $\mathrm{KG}(F G)=2$ if and only if $p=2$ and a 2-Sylow subgroup of $G$ is (isomorphic to) $C(2)^{2}$.

3) Otherwise both the width of $L_{F G}$ and consequently $\operatorname{KG}(F G)$ are undefined. Moreover, if $F$ is countable, then there exists a superdecomposable pure-injective module over FG. 
Proof. We have already mentioned that the ring $F G$ is of finite representation type if and only if a $p$-Sylow subgroup $S$ of $G$ is cyclic. This proves 1).

Therefore we may assume that a $p$-Sylow subgroup $S$ of $G$ is non-cyclic. If $p>2$ then (see Fact 4.2) FS is a wild algebra, in particular $\mathrm{wd}\left(L_{F S}\right)$ is undefined. By Proposition 4.7, $\operatorname{wd}\left(L_{F G}\right)$ is undefined.

Thus we may assume that $p=2$. If $S$ is isomorphic to $C(2)^{2}$, then, by Corollary $4.10, \operatorname{KG}(F G)=2$. Consider the other possibilities given by Fact 4.4. If $S$ has $C(4) \times C(2)$ or $C(2)^{3}$ as a factor group, then (by Fact 4.5) $F S$ is a wild algebra. As above, we obtain that $\operatorname{wd}\left(L_{F S}\right)=\infty$, hence $\operatorname{wd}\left(L_{F G}\right)=\infty$.

It remains to consider the case, when $S$ is dihedral (with $m>1$ ), semidihedral, or generalized quaternion group.

As soon as we do that, and if the field $F$ is countable, the existence of a superdecomposable pure-injective module would follow from Ziegler's result (see [20, Cor. 10.14]).

If $S$ is dihedral with $m>1$ or semidihedral, then $S$ contains $D(8)$ as a subgroup, hence it suffices to prove that $F D(8)$ has no width.

Lemma 4.12. If $F$ is a field of characteristic 2 and $D(8)$ is the dihedral group, then the width of $L_{F D(8)}$ is undefined.

Proof. Recall (see Section 4) that $D(8)$ can be realized as a group with generators $g, h$ and relations $g^{2}=h^{4}=1, g h=h^{3} g$. If $X=1+h$, $Y=1+h g$, then direct calculations show that $F D(8)$ is isomorphic to the (8-dimensional) algebra $F\left\langle X, Y \mid X^{2}=Y^{2}=0,(X Y)^{2}=(Y X)^{2}\right\rangle$ (see also $[26])$.

In particular, the 5-dimensional algebra $A=F\langle X, Y| X^{2}=Y^{2}=$ $X Y X=Y X Y=0\rangle$ is a factor algebra of $F D(8)$. It is a string algebra with two bands $C=X Y^{-1}$ and $D=X Y X^{-1} Y^{-1}$ with the same first letter (see [28] for a terminology on string algebras), hence $A$ is non-domestic. It follows from [23, Cor. 3.2] that $L_{A}$ does not have width, hence the same is true for $F D(8)$.

Indeed, the obstruction to width for $A$ refers to a suitable construction amalgamating the following finite dimensional string modules: 

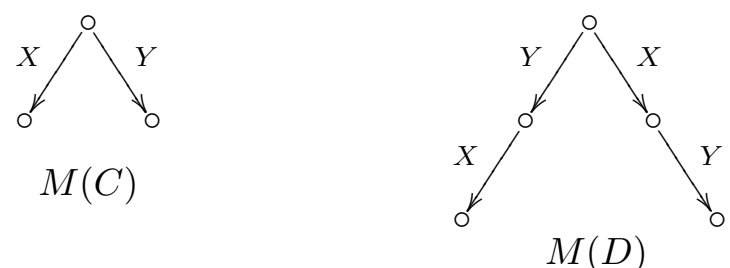

The proof in [23] uses a description of morphisms between string modules (see [7]). In Section 5 we give a more direct proof of the non-existence of width for $F D(8)$.

If $S$ is a generalized quaternion group, then $S$ contains $Q(8)$ as a subgroup. Thus, it suffices to prove that the width of $F Q(8)$ is undefined. Furthermore, by the assumption $F$ contains a primitive cubic root of unity.

Lemma 4.13. If $F$ is a field of characteristic 2 containing a primitive cubic root $\varepsilon$ of unity, and $Q(8)$ is the quaternion group, then the width of $L_{F Q(8)}$ is undefined.

Proof. Recall (see Section 4 again) that $Q(8)$ can be considered as a group with generators $g, h$ and relations $g^{4}=1, g^{2}=h^{2}$ and $g h=h^{3} g$. Here is Dade's trick. If $\varepsilon \in F$, then (see [10, p. 572]) $X=h g+\varepsilon h+\varepsilon^{2} g$ and $Y=h g+\varepsilon^{2} h+\varepsilon g$ are generators for $F Q(8)$ satisfying the following relations: $X^{2}=Y X Y, Y^{2}=X Y X, X^{2} Y=Y^{2} X=0$ and $(X Y)^{2}=(Y X)^{2}$. For instance, $F Q(8)$ has $A=F\left\langle X, Y \mid X^{2}=Y^{2}=X Y X=Y X Y=0\right\rangle$ as a factor algebra. At this point we can proceed as in the proof of the previous lemma.

Note that the only case that got unsettled in this theorem is when $F$ is a field of characteristic 2 without a primitive cubic root of unity and a 2Sylow subgroup of $G$ is a generalized quaternion group. We do believe, but unable to prove, that the width of $F G$ is undefined in this case, and clearly it suffices to prove this for $G=Q(8)$.

The following is the celebrated theorem by Brauer and Suzuki (see [4]).

Remark 4.14. If $G$ is a finite simple group, then the generalized quaternion group $Q_{m}$ cannot occur as a 2-Sylow subgroup of $G$.

Proof. We may assume that $G$ is not abelian. Then, by Glauberman $Z^{*}$ theorem (see [31, Thm. 2.14]) a 2-Sylow subgroup of $G$ contains at least two involutions (that is, elements of order 2). But (see [31, 4.2(iii)]) $Q_{m}$ contains just one involution, $g^{2}$. 
It follows that for finite simple groups Theorem 4.11 is valid without any additional restrictions.

Proposition 4.15. Let $F$ be a field of characteristic $p$, and let $G$ be a finite simple group. Then

1) $\operatorname{KG}(F G)=0$ if and only if $p$ divides $|G|$ and a $p$-Sylow subgroup of $G$ is cyclic.

2) $\mathrm{KG}(F G)=2$ if and only if $p=2$ and a 2-Sylow subgroup of $G$ is isomorphic to the Klein group $C(2)^{2}$.

3) Otherwise $\operatorname{wd}(F G)=\infty$, hence $\operatorname{KG}(F G)=\infty$.

It may be too ambitious to make a complete list of fields and finite simple groups satisfying 1) of this proposition. But it is possible to do so for $2)$. Recall that $P S L_{n}(q)$ denotes the special projective linear group over the Galois field $G F(q)$ of order $q=p^{l}$, for $p$ a prime. Note also that $P S L(2,4) \cong P S L(2,5) \cong A_{5}$.

Corollary 4.16. Let $F$ be a field and let $G$ be a finite simple group. Then the following are equivalent:

1) The Krull-Gabriel dimension of $F G$ is equal to 2.

2) The characteristic of $F$ is equal to 2 and $G \cong P S L_{2}(q)$, where $q=$ $8 k \pm 3, k \geq 1$.

Proof. By Proposition 4.15, the characteristic of $F$ must be equal to 2 . Furthermore, 1) is the same as a 2-Sylow subgroup $S$ of $G$ is isomorphic to $C(2)^{2}$. Since the Klein group is a dihedral group, we may evoke the following result by Gorenstein and Walter (see [13, Thm. 2]). A finite simple group $G$ has a dihedral 2-Sylow subgroup $S$ if and only if $G$ is isomorphic to $P S L_{2}(q)$, $q$ is odd, $q \geq 5$ or $G$ is isomorphic to $A_{7}$.

Since $\left|A_{7}\right|=\frac{7 !}{2}=2^{3} \cdot 3^{2} \cdot 5 \cdot 7$, a 2-Sylow subgroup of $A_{7}$ is isomorphic to $D_{2}=D(8)$, hence this case is ruled out. Otherwise $q=2 n+1, n \geq 2$, hence the order of $P S L_{2}(q)$ is $\frac{q\left(q^{2}-1\right)}{2}=2 n(n+1)(2 n+1)$. We have to ensure that this number is not divisible by 8 . It follows easily that $n=4 l+1$ or $n=4 l+2$ for some $l$, therefore $q=8 k \pm 3, k \geq 1$. Then $\left|P S L_{2}(q)\right|=$ $2^{2}(2 k \pm 1)(4 k \pm 1)(8 k \pm 3)$. Thus a 2-Sylow subgroup of $P S L_{2}(q)$ has order 4 , hence (by [13] again) is isomorphic to $D_{1}=C(2)^{2}$, as desired.

\section{Obstruction to the Width}

In this section we give a general construction which is very useful in proving that a particular algebra has no width 
But first we recall some facts about finitely presented (f.p.) and pureinjective modules. If $M$ is a module and $m \in M$ then the $p p$-type of $m$ in $M, p p_{M}(m)$ is the set of all pp-formulae $\varphi(x)$ such that $M=\varphi(m)$. A pp-type $p$ is said to be finitely generated, if there exists a formula $\varphi \in p$ such that $\varphi \rightarrow \psi$ for every $\psi \in p$. In this case we say that $\varphi$ generates $p$. For instance, if $M$ is a f.p. module, then the pp-type of any element $m \in M$ is finitely generated. Indeed, if $(M, m)$ is a free realization of a pp-formula $\varphi$ (see Section 2), then $\varphi$ generates $p p_{M}(m)$.

It is easily seen that, if $(M, m)$ and $(N, n)$ are pointed modules and $f$ : $M \rightarrow N$ is a pointed morphism, then $p p_{M}(m) \subseteq p p_{N}(n)$. If $M$ is f.p., the the converse is also true.

Fact 5.1. [20, Prop. 8.3] Let $(M, m)$ and $(N, n)$ be finitely presented pointed modules such that $p p_{M}(m) \subseteq p p_{N}(n)$. Then there exists a pointed morphism from $M$ to $N$.

Thus, if $(M, m)$ is a free realization of a pp-formula $\varphi$ and $(N, n)$ is a free realization of $\psi$, then there is a pointed morphism $M \rightarrow N$ if and only if $\psi \rightarrow \varphi$.

Let $M$ be an indecomposable pure-injective module, $S=\operatorname{End}(M)$. Then $S$ is a local ring, hence the set of all non-invertible elements of $S$ form a (unique) maximal ideal of $S, \operatorname{Jac}(S)$, the Jacobson radical of $S$.

Fact 5.2. [20, Thm. 4.27] Let $M$ be an indecomposable pure-injective module, $0 \neq m \in M$ and $f \in S=\operatorname{End}(M)$. Then $f \in \operatorname{Jac}(S)$ if and only if $p p_{M}(m) \subset p p_{M}(f(m))$. In particular, $m$ is not a sum of elements with strictly bigger pp-types.

Let $\mathrm{PE}(M)$ denote the pure injective envelope of a given module $M$, see [20, Ch. 4].

Fact 5.3. [15, p. 535] Let $M$ be a finitely presented module. Then $\operatorname{PE}(M)$ is indecomposable if and only if $M$ has a local endomorphism ring.

Thus the following lemma is similar to Fact 5.2.

Lemma 5.4. Let $M$ be a finitely presented module with a local endomorphism ring $S$. If $f \in S$ is such that $f(m)=m$ for some $0 \neq m \in M$, then $f$ is an automorphism. In particular, $m$ is not a sum of elements with strictly bigger pp-types. 
Proof. Since $M$ is a pure submodule of $\operatorname{PE}(M)$, we can extend $f$ to an endomorphism $g$ of $\mathrm{PE}(M)$. By Fact 5.3, $\mathrm{PE}(M)$ is an indecomposable module. Since $g(m)=m$, Fact 5.2 implies that $g$ is an automorphism of $\mathrm{PE}(M)$. Then (by the same fact) $g$ preserves pp-types of elements, hence $f=\left.g\right|_{M}$ is a pure embedding. Since $M$ is f.p., $f$ splits. But $M$ is indecomposable, hence $f$ is onto.

Suppose that $m=m^{\prime}+m^{\prime \prime}$, where the pp-types of $m^{\prime}$ and $m^{\prime \prime}$ in $M$ are strictly bigger than that of $m$. Since $M$ is f.p., there are $g, h \in \operatorname{End}(M)$ such that $g(m)=m^{\prime}$ and $h(m)=m^{\prime \prime}$. Then $(g+h)(m)=m$, hence (by what we have already proved) $g+h$ is an automorphism of $M$. Since $\operatorname{End}(M)$ is a local ring, either $g$ or $h$ is invertible, a contradiction.

The following lemma gives a useful criterion when two f.p. pointed modules are isomorphic.

Lemma 5.5. Let $(M, m),(N, n), m, n \neq 0$, be pointed finitely presented modules with local endomorphism rings. Suppose that there exist pointed morphisms $f: M \rightarrow N$ and $g: N \rightarrow M$. Then $M \cong N$.

Proof. Note that $g f$ is an endomorphism of $M$ such that $g f(m)=m$. By Lemma 5.4, $g f$ is an automorphism of $M$, therefore (multiplying by $(g f)^{-1}$ on the left) we may assume that $g f=1_{M}$. Then $f$ is a monomorphism and $N=\operatorname{im}(f) \oplus \operatorname{ker}(g)$. Since $N$ is indecomposable and $\operatorname{im}(f) \neq 0$, we conclude that $\operatorname{ker}(g)=0$, hence $f$ is onto.

Now we are in a position to present a general criterion to nonexistence of width.

Proposition 5.6. Let $R$ be a ring. Suppose that $\left(M_{q}, m_{q}\right), q \in \mathbb{Q}^{+}$and $\left(N_{t}, n_{t}\right), t \in \mathbb{Q}^{-}$are pointed finitely presented modules with local endomorphism rings such that the following conditions hold:

1) for all $0<q<q^{\prime}$ there is a pointed morphism $M_{q} \rightarrow M_{q^{\prime}}$ but the (pointed) modules $M_{q}$ and $M_{q^{\prime}}$ are not isomorphic;

2) for all $t<t^{\prime}<0$ there is a pointed morphism $N_{t^{\prime}} \rightarrow N_{t}$, but the (pointed) modules $N_{t^{\prime}}$ and $N_{t}$ are not isomorphic;

3) for all $t<0<q$ the pushout $\left(M_{t q}, m_{t q}\right)$ of $\left(N_{t}, n_{t}\right)$ and $\left(M_{q}, m_{q}\right)$ has a local endomorphism ring;

4) for all $t<0<q, q^{\prime}, q \neq q^{\prime}$, the (pointed) modules $M_{t q}$ and $M_{t q^{\prime}}$ are not isomorphic, and for all $t, t^{\prime}<0<q, t \neq t^{\prime}$, the (pointed) modules $M_{t q}$ and $M_{t^{\prime} q}$ are not isomorphic; 
5) if $t<0<q$ then there is no pointed morphism between $\left(N_{t}, n_{t}\right)$ and $\left(M_{q}, m_{q}\right)$ (in either direction).

Then the lattice $L_{R}$ of all pp-formulae over $R$ does not have width.

Proof. Let $\left(M_{q}, m_{q}\right), q \in \mathbb{Q}^{+}$be a free realization of a pp-formula $\varphi_{q}$. If $0<q<q^{\prime}$, then by 1) there is a pointed morphism $M_{q} \rightarrow M_{q^{\prime}}$. Since $\left(M_{q^{\prime}}, m_{q^{\prime}}\right)$ is a free realization of $\varphi_{q^{\prime}}$, we conclude that $\varphi_{q^{\prime}} \rightarrow \varphi_{q}$. On the other hand by 1) the (pointed) modules $M_{q^{\prime}}$ and $M_{q}$ are not isomorphic. Therefore, by Lemma 5.5, there is no pointed morphism from $M_{q^{\prime}}$ to $M_{q}$. It follows that $\varphi_{q}$ does not imply $\varphi_{q^{\prime}}$, hence $\varphi_{q^{\prime}}<\varphi_{q}$. In particular all the formulae $\varphi_{q}, q \in \mathbb{Q}^{+}$are different and form a chain $L^{+}$in $L_{R}$ isomorphic to $(\mathbb{Q}, \leq)$.

Similarly, using 2), if $\left(N_{t}, n_{t}\right), t \in \mathbb{Q}^{-}$is a free realization of a pp-formula $\psi_{t}$, then for all $t<t^{\prime}<0$ we obtain $\psi_{t}<\psi_{t^{\prime}}$. These formulae also form a chain $L^{-}$in $L_{R}$ isomorphic to $(\mathbb{Q}, \leq)$.

Let $L$ be a sublattice of $L_{R}$ generated by $L^{-} \cup L^{+}$. Since $L$ is a modular lattice generated by two chains, it is distributive. We claim that $L^{-} \cup L^{+}$ generates $L$ freely (that is, there are no relations between elements of $L$ except of those implied by the conditions that $L^{-}$and $L^{+}$are chains). Then, by [23, Cor. 3.2], it would follow that $L$, and hence $L_{R}$ does not have width.

Otherwise, by [25, Lemma 5.4], there are $\varphi_{q}>\varphi_{q^{\prime}} \in L^{+}$and $\psi_{t}>\psi_{t^{\prime}} \in$ $L^{-}$such that $\varphi_{q} \wedge \psi_{t} \leq \varphi_{q^{\prime}}+\psi_{t^{\prime}}$ (in general, some of the $\varphi^{\prime}$ 's or $\psi$ 's can be missing, but first we consider the case when all these formulas occur).

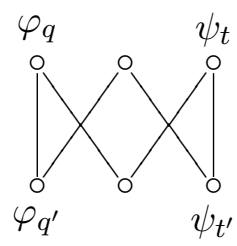

By 3) the pushout $\left(M_{t q}, m_{t q}\right)$ of $\left(N_{t}, n_{t}\right)$ and $\left(M_{q}, n_{q}\right)$ is a f.p. module with a local endomorphism ring. Clearly $\left(M_{t q}, m_{t q}\right)$ is a free realization of $\theta \doteq \varphi_{q} \wedge \psi_{t}$. Since $\theta \rightarrow \varphi_{q^{\prime}}+\psi_{t^{\prime}}$, by distributivity we obtain $\theta=\theta \wedge\left(\varphi_{q^{\prime}}+\right.$ $\left.\psi_{t^{\prime}}\right)=\left(\theta \wedge \varphi_{q^{\prime}}\right)+\left(\theta \wedge \psi_{t^{\prime}}\right)$. Thus $m_{q t}=m+n$ such that $M_{q t} \models\left(\theta \wedge \varphi_{q^{\prime}}\right)(m)$ and $M_{q t}=\left(\theta \wedge \psi_{t^{\prime}}\right)(n)$. By Lemma 5.4, $m_{q t}$ cannot be a sum of elements with strictly bigger pp-types. It follows that either $\theta$ is equivalent to $\theta \wedge \varphi_{q^{\prime}}$, whence $\theta \rightarrow \varphi_{q^{\prime}}$, or $\theta$ is equivalent to $\theta \wedge \psi_{t^{\prime}}$, whence $\theta \rightarrow \psi_{t^{\prime}}$.

By symmetry we may assume that $\theta \rightarrow \varphi_{q^{\prime}}$, that is, $\varphi_{q} \wedge \psi_{t} \rightarrow \varphi_{q^{\prime}}$. Since $\varphi_{q^{\prime}}<\varphi_{q}$, it follows easily that $\varphi_{q} \wedge \psi_{t}$ is equivalent to $\varphi_{q^{\prime}} \wedge \psi_{t}$. Then, by Remark 5.1, there is a pointed morphism from $M_{t q}$ to $M_{t q^{\prime}}$, and there is a 
pointed morphism from $M_{t q^{\prime}}$ to $M_{t q}$. By Lemma 5.5 we conclude that the pointed modules $M_{t q}$ and $M_{t q^{\prime}}$ are isomorphic, a contradiction.

It remains to consider the case when (at least) one of $\varphi_{q}$ or $\psi_{t}$ is missing, say $\varphi_{q} \leq \psi_{t^{\prime}}$. This case can be handled similarly, but we have to use 5 ).

Note that (by Corollary 3.10), this proposition remains true if we point (instead of elements) tuples of any length $k$.

We can use Proposition 5.6 to give a direct proof of the argument sketched in Section 4 to show Lemmas 4.12 and 4.13. Indeed, let $W^{+}$be the set of all words in letters $C$ and $D$ with $D$ as a first letter. We may consider $W^{+}$as a set of (indecomposable) string modules pointed at the leftmost element. Here is a typical example (a pointed element is shown by a bullet):

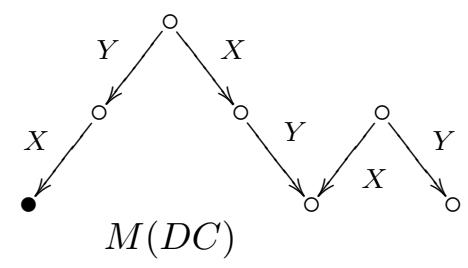

By [29, Prop. 6.2] $W^{+}$contains a countable dense chain $\left(M_{q}, m_{q}\right), q \in \mathbb{Q}^{+}$ of non-isomorphic pointed modules, such that for all $0<q<q^{\prime}$ there is a natural pointed morphism from $M_{q}$ to $M_{q^{\prime}}$. Moreover, we can choose the $M_{q}$ of (pairwise) different $F$-dimensions. Thus the modules $\left(M_{q}, m_{q}\right)$ satisfy 1) of Proposition 5.6.

Similarly let $W^{-}$be the set of all words in letters $C$ and $D$ that end by $C$. We may consider $W^{-}$as a set of string modules pointed at the rightmost element. Arguing as above, we obtain a chain of pointed modules $\left(N_{t}, n_{t}\right)$, $t \in \mathbb{Q}^{-}$of different $F$-dimensions satisfying 2) of Proposition 5.6. Clearly the pushout of $\left(N_{t}, n_{t}\right)$ and $\left(M_{q}, m_{q}\right)$ is a string module. Therefore this module is indecomposable with a local endomorphism ring. Here is an example:

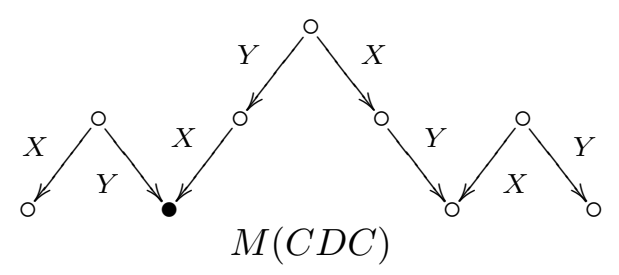

Furthermore, comparing dimensions, we see that 4) of Proposition 5.6 is also satisfied.

Concerning 5) note that $\varphi_{q}$ (the formula that generates the pp-type of $m_{q}$ in $M_{q}$ ) implies $X \mid x$ but does not imply $Y \mid x$ for every $q \in \mathbb{Q}^{+}$. Similarly $\psi_{t}$ 
(the formula that generates the pp-type of $n_{t}$ in $N_{t}$ ) implies $Y \mid x$ but does not imply $X \mid x$ for every $t \in \mathbb{Q}^{-}$. It follows (see Remark 5.1) that there is no pointed morphism between $\left(N_{t}, n_{t}\right)$ and $\left(M_{q}, n_{q}\right)$ (in either direction).

\section{REFERENCES}

[1] M. Auslander, Coherent functors, pp. 189-231 in: Proc. Conf. Categorical Algebra (La Jolla, Calif., 1965), Springer, 1966.

[2] D. Baer, Homological properties of wild hereditary Artin algebras. Representation theory, I, pp. 1-12 in: Lecture Notes in Math., 1177, Springer, 1986.

[3] V.M. Bondarenko, Yu.A. Drozd, The representation type of finite groups, J. Soviet Math., 20 (1982), 2515-2528.

[4] R. Brauer, M. Suzuki, On finite groups of even order whose 2-Sylow subgroup is a quaternion group, Proc. Nat. Acad. Sci. USA, 45 (1959), 1757-1759.

[5] K. Burke, M. Prest, The Ziegler and Zariski spectra of some domestic string algebras, Algebr. Represent. Theory, 5 (2002), 211-234.

[6] M.C.R. Butler, C.M. Ringel, Auslander-Reiten sequences with few middle terms and applications to string algebras, Comm. Algebra, 15 (1987), 145-179.

[7] W.W. Crawley-Boevey, Maps between representations of zero-relation algebras, J. Algebra, 126 (1989), 259-263.

[8] C. Curtis, I. Reiner, Representation theory of finite groups and associative algebras, Wiley, 1962.

[9] C. Curtis, I. Reiner, Methods of Representation Theory with Applications to Finite Groups and Orders, I. Wiley, 1981.

[10] E.C. Dade, Une extension de la théorie de Hall et Higman, J. Algebra, 20 (1972), 570-609.

[11] W. Geigle, The Krull-Gabriel dimension of the representation theory of a tame hereditary Artin algebra and applications to the structure of exact sequences, Manuscripta Math., 54 (1985), 83-106.

[12] D. Gorenstein, Finite Groups. Chelsea Publishing Company, 1980.

[13] D. Gorenstein, J.H. Walker, The characterization of finite groups with dihedral Sylow 2-subgroup. I, J. Algebra, 2 (1965), 85-151.

[14] I. Herzog, The endomorphism ring of a localized coherent functor, J. Algebra, 191 (1997), 416-426.

[15] I. Herzog, The Ziegler spectrum of a locally coherent Grothendieck category, Proc. London Math. Soc. (3), 74 (1997), 503-558.

[16] Ch. Jensen, H. Lenzing, Model-Theoretic Algebra with Particular Emphasis on Fields, Rings, Modules. Algebra, Logic and Applications, 2, Gordon and Breach Science Publishers, 1989.

[17] H. Krause, Generic modules over Artin algebras, Proc. London Math. Soc. (3), 76 (1998), 276-306.

[18] H. Krause, The Spectrum of a Module Category, Mem. Amer. Math. Soc., 149 (707), 2001. 
[19] A. Marcja, M. Prest, C. Toffalori, The stability classification for abelian-by-finite groups and modules over a group rings, J. London Math. Soc. (2), 47 (1993), 212226.

[20] M. Prest, Model Theory and Modules, London Math. Soc. Lecture Note Series, 130, Cambridge University Press, 1987.

[21] M. Prest, Morphisms between finitely presented modules and infinite-dimensional representations, pp. 447-455 in: CMS Conf. Proc., 24, Amer. Math. Soc., 1998.

[22] M. Prest, G. Puninski, Krull-Gabriel dimension of 1-domestic string algebras, Algebr. Repres. Theory, to appear.

[23] G. Puninski, Superdecomposable pure-injective modules exist over some string algebras, Proc. Amer. Math. Soc., 132 (2004), 1891-1898.

[24] G. Puninski, Krull-Gabriel dimension of a 1-domestic string algebra is finite, in preparation.

[25] G. Puninski, V. Puninskaya, C. Toffalori, Superdecomposable pure-injective modules over integral group rings, J. London Math. Soc. (2), 73 (2006), 48-64.

[26] C.M. Ringel, The indecomposable representations of the dihedral 2-groups, Math. Ann., 214 (1975), 19-34.

[27] C.M. Ringel, Tame Algebras and Integral Quadratic Forms, Lecture Notes in Mathematics, 1099, Springer, 1984.

[28] J. Schröer, On Krull-Gabriel dimension of an algebra, Math. Z., 233 (2000), 287-303.

[29] J. Schröer, On the infinite radical of a module category, Proc. London Math. Soc. (3), 81 (2000), 651-674.

[30] D. Simson, Linear Representations of Partially Ordered Sets and Vector Space Categories. Algebra, Logic and Applications, 4, Gordon and Breach Science Publishers, 1992.

[31] M. Suzuki, Group Theory, II. Grundlehren der Mathematischen Wissenschaften, 248, Springer, 1986.

[32] M. Ziegler, Model theory of modules, Ann. Pure Appl. Logic, 26 (1984), 149-213.

Department of Mathematics, University of Manchester, Lamb Building, Booth Street East, M13 9PL, Manchester, UK

E-mail address: gpuninski@maths.man.ac.uk

Department of Mathematics and Informatics, University di CAmerino, Via Madonna delle Carceri 9, 62032, Camerino, Italy

E-mail address: vera.puninskaya@unicam.it

E-mail address: carlo.toffalori@unicam.it 